\title{
Doubly Charmed Baryon Production in Hadronic Experiments
}

\author{
Berezhnoy A.V., Kiselev V.V., Likhoded A.K., Onishchenko A.I. \\ State Research Center of Russia "Institute for High Energy Physics" \\ Protvino, Moscow region, 142284 Russia \\ Fax: +7-095-2302337 \\ E-mail: kiselev@mx.ihep.su
}

\begin{abstract}
In the leading order of perturbative QCD one calculates the total and differential cross-sections for the hadronic production of doubly charmed baryons $\Xi_{c c}$ and $\Xi_{c c}^{*}$ in different experiments. The experimental evaluation of crosssections for the $J / \Psi+D+\bar{D}$ production would allow one to decrease the uncertainty in the determination of cross-sections for the doubly charmed baryons due to the choice of $\alpha_{s}$ and $m_{c}$. One shows that in the HERA-B and E781 experiments with fixed tagets the suppression of the $\Xi_{c c}$ and $\Xi_{c c}^{*}$ production to the yield of $c \bar{c}$-pairs is the value of the order of $10^{-6}-10^{-5}$, whereas at the TEVATRON and LHC colliders it is about $10^{-4}-10^{-3}$. In the E781 experiment the observation of $\Xi_{c c}$ and $\Xi_{c c}^{*}$ is practically unpossible. At the HERA-B and TEVATRON facilities one can expect $10^{5}$ events with the double charm, and at LHC one has about $10^{9}$ ones.
\end{abstract}

PACS numbers: 14.20.Lq, 13.60.Rj, 12.38.Bx, 13.30.-a 


\section{INTRODUCTION}

Recent years are marked by a rapid increase of charmed particles observed in modern experiments. So, the study of about $10^{6}$ charmed particles is expected at fixed target FNAL facilities of E831 and E781. An increase of this value by two orders of magnitude is proposed in experiments of next generation. Along with standard problems of CP-violation in the charmed quark sector and a measuring of rare decays etc., an investigation of processes with more than one $c \bar{c}$-pair production becomes actual. The production of additional $c \bar{c}$-pair strongly decreases a value of cross-section for such processes. This fact must be especially taken into account in fixed target experiments, where the quark-partonic luminosities are strongly suppressed in the region of heavy mass production.

An interesting process of the mentioned kind is the doubly charmed baryon production. The doubly charmed $\Xi_{c c}^{(*)}$-baryon represents an absolutely new type of objects in comparison with the ordinary baryons containing light quarks only. The basic state of such baryon is analogous to a $(\bar{Q} q)$-meson, which contains one heavy antiquark $\bar{Q}$ and one light quark $q$. In the doubly heavy baryon the role of heavy antiquark is played by the (cc)-diquark, which is in antitriplet color-state [1]. It has a small size in comparison with the scale of the light quark confinement.

The spectrum of (ccq)-system states has to differ essentially from the heavy meson spectra, because the composed $(c c)$-diquark has a set of the excited states (for example, $2 S$ and $2 P$ ) in contrast to the heavy quark. The energy of diquark excitation is twice less than the excitation energy of light quark bound with the diquark. So, the representation on the compact diquark

can be straightforwardly connected with the level structure of doubly heavy 
baryon円.

Another interesting aspect of the doubly charmed baryon researches is a production mechanism. The $(c c q)$-baryon production was discussed in the number of papers [4]- [7]. The main problem of calculations is reduced to an evaluation of the production cross section for the diquark in the antitriplet color state. One assumes further that the $(c c)$-diquark nonperturbatively transforms into the $(c c q)$-baryon with a probability close to unit. The hadronic production of diquark is subdivided into two parts. The first stage is the hard production of two $(c \bar{c})$-pairs in the processes of $g g \rightarrow c \bar{c} c \bar{c}$ and $q \bar{q} \rightarrow c \bar{c} c \bar{c}$, which are described by the Feynman diagrams of the fourth order over the $\alpha_{s}$ coupling constant [8]. The second step is the nonperturbative fusion of two $c$-quarks with a small relative momentum into the $(c c)$-diquark. For the $S$-wave states, this process is characterized by the radial wave function at the origin, $R(0)$.

The main difference between the existing evaluations of the doubly charmed baryon cross section consists in the methods used for the hard subprocess calculation. In paper [9] a part of diagrams connected with the $c$ fragmentation into the $(c c)$-diquark is only used instead of the complete set of diagrams. As was shown in paper [6] this estimation is not absolutely

\footnotetext{
* Our estimates of diquark mass in the Martin [2] potential with taking into account the color factor for antitriplet state of the quark pair and using the results of heavy quark effective theory [3], give the value of $M\left(\Xi_{c c}^{(*)}\right)=3.615 \pm 0.035 \mathrm{GeV}$ (without taking into account a spin dependent interaction). The mass shift of vector diquark is determined by the formula $\delta M \simeq \frac{1}{2}\left|R_{c c}(0)\right|^{2} /\left|R_{\psi}(0)\right|^{2}\left(M_{\psi}-M_{\eta_{c}}\right) / 4 \simeq 5 \mathrm{MeV}$. The splitting between $\Xi_{c c}$ and $\Xi_{c c}^{*}$ is equal to $\Delta M\left(\Xi_{c c}^{(*)}\right) \simeq \frac{3}{4} \Delta M\left(D^{(*)}\right) \simeq 108 \mathrm{MeV}$, so $M\left(\Xi_{c c}\right)=3.584 \pm 0.035 \mathrm{GeV}, M\left(\Xi_{c c}^{*}\right)=3.638 \pm 0.035$ $\mathrm{GeV}$. The diquark size $r_{c c} \sim 0.5 \mathrm{fm}$ is close to that of $J / \Psi$.
} 
correct, because it becomes true only at $p_{T}>35 \mathrm{GeV}$, where the fragmentation mechanism is dominant. In other kinematical regions the application of fragmentational approximation is not justified and it leads to wrong results, especially at $\sqrt{\hat{s}}$ being not much greater than $p_{T}^{\text {min }}$.

In the framework of fragmentation model the account for the leading logarithm corrections can be performed in a simple way. However, in the framework of calculations for the complete set of diagrams in the $\alpha_{s}^{4}$-order the regime of fragmetation is dominant only in the restricted region of kinematical variables, $p_{T}>p_{T}^{\text {min }}, s>s^{\text {min }}$, wherein the procedure for the taking into account the evolution of fragmentation functions does not cause some difficulties. But the account for the emission of additional hard gluon beyond the mentioned region requires the calculations in the next $\alpha_{s}^{5}$-order of perturbative QCD, so that the number of diagrams increases drastically, and there are no large $\operatorname{logs}$ like $\log \left(p_{T}^{2} / m^{2}\right)$ or $\log \left(s / m^{2}\right)$ in the dominant kinematical domain. So, following refs. [4- [7], in this paper we are restricted by the Born approximation.

However, even after taking into account the complete set of diagrams essential uncertainties in the estimations of the $(c c q)$-baryon production remain. The basic parameters determining these uncertainties are the values of $\alpha_{s}, m_{c}$ and $R_{c c}(0)$. In addition, it is not clear, to what extent the hypothesis on the hadronization of $(c c)$-diquark into the $(c c q)$-baryon with the unit probability is correct or not. The matter is that the interaction between the diquark and gluons is not suppressed in contrast to the $(c \bar{c})$-pair production in the color singlet state, when the quarkonium dissociation supposes exchange with the quark-gluonic sea by two hard gluons with virtualities, which are greater than the inverse size of quarkonium.

A decrease of the uncertainty in the $(c c q)$-baryon cross-section would be possible by means of comparing the process of baryon production with the 
analogous process of $J / \Psi+D \bar{D}$ production. The latter is described by practically the same diagrams of fourth order with the well-known wave function of $J / \Psi$ at the originf.

In this way of connection to the $J / \Psi+D \bar{D}$ process one could remove the part of uncertainties, which are due to $\alpha_{s}$ and $m_{c}$ in the $(c c)$-diquark production process. In the following sections of the paper the joint cross-section calculations of these processes in $\pi^{-} p$ and $p p$ interactions are performed.

There are some papers, wherein the fragmentation model for the $J / \psi$ production at $p_{T}>5 \mathrm{GeV}$ was constructed with the account for the coloroctet contribution (see the review [10]). However, the associated production of $J / \psi+D \bar{D}$ requires the production of additional $c \bar{c}$-pair, so that the production mechanism is evidently the other one, and the $J / \psi+D \bar{D}$ yield is only a small fraction of the inclusive $J / \psi$ production in $p p$-interactions.

For the $B_{c}+b \bar{c}$ production one has shown that the regime of fragmentation becomes dominant at $p_{T}>35 \mathrm{GeV}$. The production of $J / \psi+c \bar{c}$ is completely analogous to the associated production of $B_{c}+b \bar{c}$ (with the careful account for the identity of charmed quarks). For the $B_{c}+b \bar{c}, \Xi_{c c}+\bar{c} \bar{c}$ and $J / \psi+c \bar{c}$ production one finds the common regularity: the fragmentation regime is displaced to the region of $p_{T}>35 \mathrm{GeV}$. To convince completely, we present the corresponding figure for the gluon-gluon subprocess of $g g \rightarrow J / \psi+c \bar{c}$ at $\sqrt{\hat{s}}=100 \mathrm{GeV}$. Thus, we insist on the statement that for the associated

$\dagger$ We calculate the $J / \psi+c \bar{c}$ production and assume that the $c \bar{c}$-pair transforms to $D \bar{D}+$ some light hadrons with the probability very close to unit. So, we neglect the production of charmed baryons as well as bound states of charmonium.

$\ddagger$ The value of $\left|R_{\Psi}(0)\right|$ is determined by the width of leptonic decay, $J / \Psi \rightarrow l^{+} l^{-}$with taking into account the hard gluonic correction, so, numerically, $\left|R_{\psi}(0)\right|=\sqrt{\pi M / 3} \tilde{f}_{\psi}$, where $\tilde{f}_{\psi}=540 \mathrm{MeV}$. 
production of $J / \psi+c \bar{c}$ and $\Xi_{c c}+\bar{c} \bar{c}$ the fragmentation works at $p_{T} \gg m_{c}$.

Section II is devoted to the description of production models for the $(c c q)$ baryons and $J / \Psi+D \bar{D}$. In Section III one presents the calculation results for the production cross-section of $(c c q)$-baryons and $J / \Psi+D \bar{D}$ in the fixed target experiments E781 and HERA-B.

\section{PRODUCTION MECHANISM}

As was mentioned in Introduction, we suppose that the diquark production can be subdivided by two stages. On the first stage the production amplitude of four free quarks is calculated for the following processes

$$
\begin{aligned}
& g g \rightarrow c c \bar{c} \bar{c} \\
& q \bar{q} \rightarrow c c \bar{c} \bar{c} .
\end{aligned}
$$

The calculation technique applied in this work is analogous to that for the hadronic production of $B_{c}$ [11], but in this case the bound state is composed by two quarks [5,6] instead of the quark and antiquark.

One assumes that the binding energy in the diquark is much less than the masses of constituent quarks and, therefore, these quarks are on the mass shells. So, the quark four-momenta are related to the $\left(Q_{1} Q_{2}\right)$ diquark momentum in the following way

$$
p_{Q_{1}}=\frac{m_{Q_{1}}}{M_{\left(Q_{1} Q_{2}\right)}} P_{\left(Q_{1} Q_{2}\right)}, \quad p_{Q_{2}}=\frac{m_{Q_{2}}}{M_{\left(Q_{1} Q_{2}\right)}} P_{\left(Q_{1} Q_{2}\right)}
$$

where $M_{\left(Q_{1} Q_{2}\right)}=m_{Q_{1}}+m_{Q_{2}}$ is the diquark mass, $m_{Q_{1}}, m_{Q_{2}}$ are the quark masses.

In the given approach the diquark production is described by 36 Feynman diagrams of the leading order, corresponding to the production of four free quarks with the combining of two quarks into the color antitriplet diquark with 
the given quantum numbers over the Lorentz group. The latter procedure is performed by means of the projection operators

$\mathcal{N}(0,0)=\sqrt{\frac{2 M_{\left(Q_{1} Q_{2}\right)}}{2 m_{Q_{1}} 2 m_{Q_{2}}}} \frac{1}{\sqrt{2}}\left\{\bar{u}_{1}\left(p_{Q_{1}},+\right) \bar{u}_{2}\left(p_{Q_{2}},-\right)-\bar{u}_{1}\left(p_{Q_{1}},-\right) \bar{u}_{2}\left(p_{Q_{2}},+\right)\right\}$,

for the scalar state of diquark (the corresponding baryon is denoted as $\left.\Xi_{Q_{1} Q_{2}}^{\prime}(J=1 / 2)\right)$

$\mathcal{N}(1,-1)=\sqrt{\frac{2 M_{\left(Q_{1} Q_{2}\right)}}{2 m_{Q_{1}} 2 m_{Q_{2}}}} \bar{u}_{1}\left(p_{Q_{1}},-\right) \bar{u}_{2}\left(p_{Q_{2}},-\right)$,

$\mathcal{N}(1,0)=\sqrt{\frac{2 M_{\left(Q_{1} Q_{2}\right)}}{2 m_{Q_{1}} 2 m_{Q_{2}}}} \frac{1}{\sqrt{2}}\left\{\bar{u}_{1}\left(p_{Q_{1}},+\right) \bar{u}_{2}\left(p_{Q_{2}},-\right)+\bar{u}_{1}\left(p_{Q_{1}},-\right) \bar{u}_{2}\left(p_{Q_{2}},+\right)\right\}$,

$\mathcal{N}(1,+1)=\sqrt{\frac{2 M_{\left(Q_{1} Q_{2}\right)}}{2 m_{Q_{1}} 2 m_{Q_{2}}}} \bar{u}_{1}\left(p_{Q_{1}},+\right) \bar{u}_{2}\left(p_{Q_{2}},+\right)$

for the vector state of diquark (the baryons are denoted as $\Xi_{Q_{1} Q_{2}}(J=1 / 2)$ and $\left.\Xi_{Q_{1} Q_{2}}^{*}(J=3 / 2)\right)$.

To produce the quarks, composing the diquark in the $\overline{3}_{c}$ state, one has to introduce the color wave function as $\varepsilon_{i j k} / \sqrt{2}$, into the diquark production vertex, so that $i=1,2,3$ is the color index of the first quark, $j$ is that of the second one, and $k$ is the color index of diquark.

The diquark production amplitude $A_{k}^{S s_{z}}$ is expressed through the amplitude $T_{k}^{S s_{z}}\left(p_{i}\right)$ for the free quark production in kinematics (3)

$$
A_{k}^{S s_{z}}=\frac{R_{Q_{1} Q_{2}}(0)}{\sqrt{4 \pi}} T_{k}^{S s_{z}}\left(p_{i}\right)
$$

where $R_{Q_{1} Q_{2}}(0)$ is the diquark radial wave function at the origin, $k$ is the color state of diquark, $S$ and $s_{z}$ are the diquark spin and diquark spin projection on the $z$-axis, correspondingly.

In the numerical calculation giving the results, which will be discussed in the next Section, one supposes the following values of parameters

$$
\begin{aligned}
& \alpha_{s}=0.2, \\
& m_{c}=1.7 \mathrm{GeV}, \\
& R_{c c(1 S)}(0)=0.601 \mathrm{GeV}^{3 / 2},
\end{aligned}
$$


where the value of $R_{c c}(0)$ has been calculated by means of numerical solution of the Schrödinger equation with the Martin potential [12], multiplied by the $1 / 2$ factor caused by the color antitriplet state of quarks instead of the singlet one.

To calculate the production cross-section of diquarks composed of two $c$ quarks, one has to account for their identity. One can easily find, that the antisymmetrization over the identical fermions leads to the scalar diquark amplitude equal to zero, and it results in the amplitude of the vector $(c c)$ diquark production being obtained by the substituting of equal masses in the production amplitude of vector diquark composed of two quarks with the different flavors, and taking into account the $1 / 2$ factor for the identical quarks and antiquarks.

In this work one supposes that the produced diquark forms the baryon with the unit probability by catching up the light quark from the quarkantiquark sea at small $p_{T}$ or having the fragmentation into the baryon at large $p_{T}$.

The typical diagrams of fourth order describing the processes (1) and (2) with the binding of $c c$-pair into the diquark are shown in Fig. 1. One can subdivide them into two groups. The first group contains the diagrams of fragmentation type, wherein the $(c \bar{c})$-pair emits another one. The second group corresponds to the independent dissociation of gluons into the $(c \bar{c})$-pairs with the following fusion into the diquark. The diagrams of second group belong to the recombination type. As was mentioned above, the authors of some papers restricted themselves by the consideration of fragmentation diagrams, only. In this way they reduced the cross-section formulae to the $(c \bar{c})$-pair production cross-section multiplied by the fragmentation function of $c$-quark into the $(c c)$-diquark. As was shown in [6], the latter approach is correct only 
under the two following conditions: $M_{(c c)}^{2} \ll \hat{s}$ and $p_{T} \gg M_{(c c)}$. In other kinematical regions, the contribution of recombination diagrams dominates.

The typical value of $p_{T}$, wherefrom the fragmentation begins to dominate, is $p_{T}>35 \mathrm{GeV}$. It is clear, that at realistic $p_{T}$ one has to take into account all contributions including the recombination one. For the first time, the complete set of diagrams was taken into account in [6] and, after that, in [7]. In the both papers the calculations are performed only for the gluon-gluon production, which is a rather good approximation at collider energies. For the fixed target experiments the value of total energy strongly decreases, and, hence, the values of energy in the subprocesses (1) and (2) decrease too.

The contribution of quark-antiquark annihilation becomes essential at fixed target energies, especially for the processes with initial valent antiquarks. In the following consideration one allows for the quark-antiquark annihilation into four free charmed quarks in the estimation of yield for the doubly charmed baryon. To our knowledge, the corresponding calculations for the diquarkproduction were not yet performed early, so, they are carried out here for the first time.

\section{DOUBLY CHARMED BARYON PRODUCTION IN FIXED TARGET EXPERIMENTS}

The applied method of calculations is the same as in our previous works [6,11. One calculates the complete set of diagrams in the fourth order over the strong coupling constant for the Born amplitude of process under consideration.

The calculation results for the total cross-section of the diquarkproduction subprocesses versus the total energy are shown in Figs. 2 and

3 for the given values of $\alpha_{s}, m_{c}$ and $R_{(c c)}(0)$. These dependencies can be 
approximately described by the following expressions

$$
\begin{aligned}
& \hat{\sigma}_{g g}^{(c c)}=213 \cdot\left(1-\frac{4 m_{c}}{\sqrt{\hat{s}}}\right)^{1.9}\left(\frac{4 m_{c}}{\sqrt{\hat{s}}}\right)^{1.35} \mathrm{pb} \\
& \hat{\sigma}_{q \bar{q}}^{(c c)}=206 \cdot\left(1-\frac{4 m_{c}}{\sqrt{\hat{s}}}\right)^{1.8}\left(\frac{4 m_{c}}{\sqrt{\hat{s}}}\right)^{2.9} \mathrm{pb} .
\end{aligned}
$$

One has to mention that the numerical coefficients depend on the model parameters, so that $\hat{\sigma} \sim \alpha_{s}^{4}|R(0)|^{2} / m_{c}^{5}$.

As was mentioned in the Introduction, the production of $J / \Psi$ in the subprocesses of $g g \rightarrow J / \Psi+c \bar{c}$ and $q \bar{q} \rightarrow J / \Psi+c \bar{c}$ is also calculated in this work. The numerical results of such consideration are shown in Figs. 2 and 3 . The parameterization of these results versus the energy $\sqrt{\hat{s}}$ are presented below

$$
\begin{aligned}
& \hat{\sigma}_{g g}^{J / \Psi}=518 .\left(1-\frac{4 m_{c}}{\sqrt{\hat{s}}}\right)^{3.0}\left(\frac{4 m_{c}}{\sqrt{\hat{s}}}\right)^{1.45} \mathrm{pb}, \\
& \hat{\sigma}_{q \bar{q}}^{J / \Psi}=699 \cdot\left(1-\frac{4 m_{c}}{\sqrt{\hat{s}}}\right)^{1.9}\left(\frac{4 m_{c}}{\sqrt{\hat{s}}}\right)^{2.97} \mathrm{pb} .
\end{aligned}
$$

As well as for the $B_{c}+b \bar{c}, \Xi_{c c}+\bar{c} \bar{c}$ production, one finds the following regularity for the $J / \psi+c \bar{c}$ production: the fragmentation regime is displaced to the region of $p_{T}>35 \mathrm{GeV}$. The latter fact can be certainly observed in the figure for the gluon-gluon subprocess of $g g \rightarrow J / \psi+c \bar{c}$ at $\sqrt{\hat{s}}=100 \mathrm{GeV}$ (Fig. 四). Thus, for the associated production of $J / \psi+c \bar{c}$ and $\Xi_{c c}+\bar{c} \bar{c}$ the fragmentation works at $p_{T} \gg m_{c}$.

These formulae quite accurately reconstruct the results of precise calculations at $\sqrt{\hat{s}}<150 \mathrm{GeV}$, and that is why they can be used for the approximate estimation of total hadronic production cross-section for the $(c c)$-diquark and $J / \Psi$ by means of their convolution with the partonic distributions as below

$$
\sigma=\sum_{i, j} \int d x_{1} d x_{2} f_{i / A}\left(x_{1}, \mu\right) f_{j / B}\left(x_{2}, \mu\right) \hat{\sigma}
$$

where $f_{i / A}(x, \mu)$ is the distribution of $i$-kind parton in the $A$-hadron. The parton distributions used for the proton are the CTEQ4 parameterizations [12], and those of used for the $\pi^{-}$-meson are the Hpdf ones [13]. In both these 
cases the virtuality scale is fixed at $10 \mathrm{GeV}$. As for the choice of fixed scale in the structure functions, this is caused by the fact that the cross-section of subprocesses is integrated in the region of low $\hat{s}$ close to the fixed scale, so that the account of "running" scale weakly changes the estimate of $\Xi_{c c^{-}}$ baryon yield in comparison with the mentioned uncertainty of diquark model (we have found the scale-dependent variation to be at the level of $\delta \sigma / \sigma \sim 10$ $\%)$.

The total hadronic production cross-section for these processes are presented in Figs. 5 and 6 for the $\pi^{-} p$ and $p p$-interactions, correspondingly. As one can see in Figs. 5 and 6, the cross-section of $(c c)$-diquark as well as the cross-section of $J / \Psi+c \bar{c}$ are strongly suppressed at low energies in comparison with the values at the collider energies.

The ratio for the $(c c)$-diquark production and total charm production is $\sigma_{(c c)} / \sigma_{\text {charm }} \sim 10^{-4}-10^{-3}$ in the collider experiments and $\sim 10^{-6}-10^{-5}$ in the fixed target experiments. The same situation is observed for the hadronic $J / \Psi+D \bar{D}$ production. The distributions for the (ccq)-baryon and $J / \Psi+D \bar{D}$ production are shown in Figs. 010 for the $\pi^{-} p$-interaction at $35 \mathrm{GeV}$ and for the $p p$-interaction at $40 \mathrm{GeV}$, correspondingly. The rapidity distributions in Figs. 8 and 10 point to the central state of $(c c q)$-baryon production and that for $J / \Psi+D \bar{D}$.

The $p_{T}$-distributions of these processes are alike to each other also (we assume that at the given energies the $(c c)$-diquark has no fragmentational transition into the baryon, but it catch up the light quark from the quarkantiquark pair sea). One can see in the latter Figs., that the process of $J / \Psi+D \bar{D}$ production can be used to normalize the estimate of (ccq)-baryon yield, wherein the following additional uncertainties appear as

1. the unknown value of $\left|R_{(c c)}(0)\right|^{2}$, 
2. uncertainties related with the hadronization of $(c c)$-diquark.

One can see from the given estimates that in the experiments with the expected number of charmed events at the level about $10^{6}$ (for example, in the E781 experiment, where $\sqrt{s}=35 \mathrm{GeV}$ ), one has to expect about one event with the doubly charmed baryon. The situation is more promising and pleasant for the $p p$-interaction at $800 \mathrm{GeV}$ (HERA-B). The considered processes yield about $10^{5} \Xi_{c c}^{(*)}$-baryons and a close number of $J / \Psi+D \bar{D}$ in the experiment specialized for the detection of about $10^{8}$ events with the $b$-quarks.

\section{PRODUCTION OF (CCQ)-BARYON AT COLLIDERS}

As one can see in the previous Section, the observation of $\Xi_{c c}^{(*)}$-baryons presents a rather difficult problem in the experiments specialized for the study of charmed particles. As a rule, such experiments are carried out at fixed targets, so that the effective value of subprocess energy is strongly decreased. So, the relative contribution of doubly charmed baryons into the total charm yield is of the order of $10^{-6}-10^{-5}$. The production of $(c c q)$-baryons at colliders with large $p_{T}$ is more effective. In this case the cross-section is determined by the region of quark-antiquark and gluon-gluonic energy, where the threshold effect becomes negligible and the partonic luminosities are quite large at $x \sim M / \sqrt{s}$. So, the suppression factor in respect to the single production of $c \bar{c}$-pairs is much less and it is in the range of $10^{-4}-10^{-3}$.

The $p_{T}$-distributions for $\Xi_{c c}^{(*)}$ and $J / \Psi$ (which is produced with $D$ and $\bar{D}$ ) at TEVATRON and LHC are shown in Figs. 11 and 12. The rapidity cut $(|y|<1)$ is taken into account.

One can easily understand that the presented $\Xi_{c c}^{(*)}$ cross-sections are the upper estimates for the real cross-sections because of the possible dissociation of heavy diquark into the $D D$-pair. 
Further, even if the $(c c)$-diquark, being the color object, transforms into the baryon with the unit probability, one has to introduce the fragmentation function describing the hadronization of diquark into the baryon at quite large $p_{T}$ values. The simplest form of this function can be chosen by analogy with that for the heavy quark

$$
D(z) \sim \frac{1}{z} \frac{1}{\left(m_{c c}^{2}-\frac{M^{2}}{z}-\frac{m_{q}^{2}}{1-z}\right)^{2}},
$$

where $M$ is the mass of baryon $\Xi_{c c}^{(*)}, m_{c c}$ is the mass of diquark, $m_{q}$ is the mass of light quark (we suppose it to be equal to $300 \mathrm{MeV}$ ).

The $p_{T}$-distributions of doubly charmed baryon production, as those are shown in Figs. 11 and 12, are calculated with the use of (13).

One has to mention, that in the leading order over the inverse heavy quark mass, the relative yield of $\Xi_{c c}$ and $\Xi_{c c}^{*}$ is determined by the simple counting rule for the spin states, and it equals $\sigma\left(\Xi_{c c}\right): \sigma\left(\Xi_{c c}^{*}\right)=1: 2$. In this approach one does not take into account a possible difference between the fragmentation functions for the baryons with the different spins. The corresponding difference is observed in the perturbative fragmentation functions for the heavy mesons and quarkonia [14].

\section{DISCUSSION}

As we have shown on the basic of perturbative calculations for the hard production of doubly charmed diquark fragmentating in the baryon, the observation of doubly charmed baryons is a difficult problem, because the ratio

of $\sigma\left(\Xi_{c c}^{(*)}\right) / \sigma($ charm $)$ for these baryons and charmed particles yields the value of $10^{-6}-10^{-3}$ depending on the process energy. The suppression of doubly charmed baryon yield at low energies is explained by threshold effect. As one can see in accordance with Tab. 1 , about $10^{5}$ events with the production 
of $\Xi_{c c}^{(*)}$-baryons can be expected at HERA-B. Practically the same number of events at $p_{T}>5 \mathrm{GeV}$ and $|y|<1$ is expected at TEVATRON with the integrated luminosity of $100 \mathrm{pb}^{-1}$. The large luminosity and large interaction energy allow one to increase the yield of the doubly charmed baryon by $10^{4}$ times at LHC.

Under conditions of large yield of the doubly charmed baryons, the problem of their registration appears.

First of all it is interesting to estimate the lifetimes of the lightest states of $\Xi_{c c}^{++}$and $\Xi_{c c}^{+}$. The simple study of quark diagrams shows that in the decay of $\Xi_{c c}^{++}$-baryons the Pauli interference for the decay products of charmed quark and valent quark in the initial state takes place as well as in the case of $D^{+}$meson decay. In the decay of $\Xi_{c c}^{+}$the exchange by the $W$-boson between the valent quarks plays an important role as well as in the decay of $D^{0}$. Therefore we suppose that the mentioned mechanisms give the same ratio for the both baryon and $D$-meson lifetimes

$$
\tau\left(\Xi_{c c}^{+}\right) \approx 0.4 \cdot \tau\left(\Xi_{c c}^{++}\right)
$$

The presence of two charmed quark in the initial state results in following expressions

$$
\begin{aligned}
\tau\left(\Xi_{c c}^{++}\right) & \approx \frac{1}{2} \tau\left(D^{+}\right) \simeq 0.53 \mathrm{ps} \\
\tau\left(\Xi_{c c}^{+}\right) & \approx \frac{1}{2} \tau\left(D^{0}\right) \simeq 0.21 \mathrm{ps} .
\end{aligned}
$$

One can point to the important decay modes of these baryons in analogy with the case of the charmed mesons

$$
\mathrm{BR}\left(\Xi_{c c}^{++} \rightarrow K^{0(*)} \Sigma_{c}^{++(*)}\right) \approx \mathrm{BR}\left(\Xi_{c c}^{+} \rightarrow K^{0(*)}\left(\Sigma_{c}^{+(*)}+\Lambda_{c}^{+}\right)\right) \approx \mathrm{BR}\left(\Lambda_{c} \rightarrow K^{0(*)} p\right) \simeq 4 \cdot 10^{-2} .
$$

One can observe $4 \cdot 10^{3}$ events in these decay modes at HERA-B and TEVATRON without taking into account a detection efficiency. One has to 
expect the yield of $4 \cdot 10^{7}$ such decays at LHC. Among other decay modes, $\Xi_{c c}^{++} \rightarrow \pi^{+} \Xi_{c}^{+}$and $\Xi_{c c}^{+} \rightarrow \pi^{+} \Xi_{c}^{0}$ taking place with the probability of about $1 \%$, can be essential.

The excited $\Xi_{c c}^{*}$ states always decay into $\Xi_{c c}$ by the emission of $\gamma$-quanta so that the branching fraction of transition is equal to $100 \%$, since the emission of $\pi$-meson is impossible in the $\Xi_{c c}^{*}$ decay because of the small value of splitting between the basic state and the excited one, in contrast to the charmed meson decay.

In conclusion we mention another possibility to increase the yield of doubly charmed baryons in fixed target experiments. In the model of intrinsic charm 15 one assumes, that the nonperturbative admixture of exotic hybrid state $|c \bar{c} u u d\rangle$ presents in the proton along with the ordinary state $|u u d\rangle$ including three light valent quarks. The probability $P_{i c}$ of $|c \bar{c} u u d\rangle$-state is suppressed at the level of $1 \%$. The valent charmed quark from that state can recombinate with the charmed quark produced in the hard partonic process of the $(c \bar{c})$-pair production. The energy dependence for such doubly charmed baryon production repeats one for the single charmed quark production in the framework of pQCD up to the factor of exotic state suppression and the factor of fusion of two charmed quarks into the diquark, $K \sim 0.1$. This mechanism has no threshold of four quark state production in contrast to the discussed perturbative one. Therefore at low energies of fixed target experiments, where the threshold suppression of perturbative mechanism is strong, the model of intrinsic charm would yield the dominant contribution into the $\Xi_{c c}^{(*)}$ production. So, the number of events in this model would be increased by three orders of magnitude, and ratio of $\Xi_{c c}^{(*)}$ and charmed particle yields would equal $\sigma\left(\Xi_{c c}^{(*)}\right) / \sigma(\mathrm{charm}) \sim 10^{-3}$. At high energies the perturbative production is comparable with the intrinsic charm contribution. One has to note, that the $|c \bar{c} c \bar{c} u u d\rangle$-state suppressed at the level of $3 \cdot 10^{-4}$, also could 
increase the doubly charmed baryon production at low energies of hadronhadron collisions.

Thus, the observation of $\Xi_{c c}^{*}$-baryons in hadronic interactions is a quite realistic problem, whose solution opens new possibilities to research the heavy quark interactions. The observation of $\Xi_{c c}^{(*)}$-baryons at fixed target experiments [16] would allow one to investigate the contributions of different mechanisms in the doubly charmed baryon production, as the contribution of the perturbative mechanism and that of the intrinsic charm, which strongly increases the yield of these baryons.

The authors express their gratitude to A.Kulyavtsev for the discussion of problem under consideration.

This work is supported, in part, by the Russian Foundation for Basic Research, grants 96-02-18216 and 96-1596575. The work of A.V. Berezhnoy has been made possible by a fellowship of INTAS Grant 93-2492 and is carried out within the research program of International Center for Fundamental Physics in Moscow.

[1] Georgi H., Wise M.B., Phys. Lett. B243, 279 (1990);

Carone C.D., Phys. Lett. B253, 408 (1991);

Savage M.J., Wise M.B., Phys. Lett. B248, 177 (1990).

[2] Martin A., Phys. Lett. B93, 338 (1980).

[3] Neubert M., Phys. Rep. 245, 259 (1994).

[4] Falk A. et al., Phys. Rev. D49, 555 (1994).

[5] Kiselev V.V., Likhoded A.K., Shevlyagin M.V., Phys. Lett. B332, 411 (1994);

Berezhnoy A.V., Kiselev V.V., Likhoded A.K., Z. Phys. A356, 89 (1996). 
[6] Berezhnoy A.V., Kiselev V.V., Likhoded A.K., Phys. Atom. Nucl. 59, 870 (1996).

[7] Baranov S.P., Phys. Rev. D54, 3228 (1996).

[8] Barger V., Phillips R.J.N., Stange A.L., Phys. Rev. D44, 1987 (1991).

[9] Doncheski M.A., Steegborn J., Strong M.L., Phys. Rev. D53, 1247 (1996).

[10] Braaten E., Fleming S., Yuan T.Ch., preprint OHSTPY-HEP-T-96-001, (1996) [hep$\mathrm{ph} / 9602374$.

[11] Berezhnoy A.V., Likhoded A.K., Shevlyagin M.V., Yad. Fiz. 58, 730 (1995);

Berezhnoy A.V., Likhoded A.K., Yuschenko O.P., Yad. Fiz. 59, 742 (1996);

Berezhnoy A.V., Kiselev V.V., Likhoded A.K., Z. Phys. A356, 79 (1996).

[12] Lai H.L. et al., CTEQ Coll., Preprint MSUHEP-60426 (1996) hep-ph/9606399.

[13] Owens J.F., Phys. Rev. D30, 943 (1984).

[14] Chang C.-H., Chen Y.-Q., Phys. Rev. D46, 3845 (1992), D50, 6013(E) (1994);

Braaten E., Cheung K., Yuan T.C., Phys. Rev. D48, 4230 (1993)

Kiselev V.V., Likhoded A.K., Shevlyagin M.V., Z. Phys. C63, 77 (1994);

Yuan T.C., Phys. Rev. D50, 5664 (1994);

Cheung K., Yuan T.C., Phys. Rev. D53, 3591 (1996).

[15] Brodsky S.J., Vogt R., Nucl. Phys. B478, 311 (1996).

[16] Moinester M.A., Z. Phys. A355, 349 (1996);

Kaplan D.M., Kroan S., FERMILAB-Conf-94/190. 
TABLE I. The production cross-section of doubly charmed baryons at different facilities.

\begin{tabular}{|c|c|c|c|r|}
\hline \hline facility & HERA-B & E781 & TEVATRON & LHC \\
\hline total cross-section, \\
$\mathrm{nb} /$ nucleon
\end{tabular}



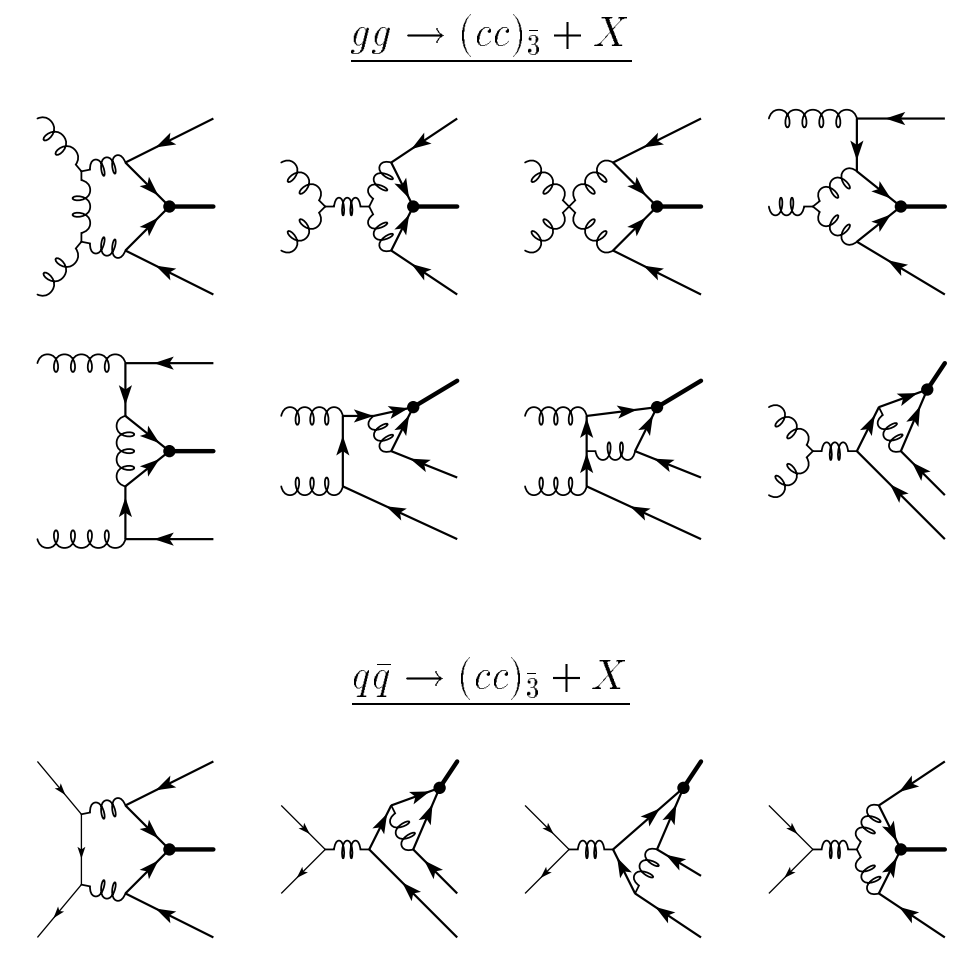

FIG. 1. The examples of diagrams for the gluon-gluon and quark-antiquark production of (cc)-diquark. The initial quarks are denoted by the thin fermion lines, the final quarks are denoted by the bold fermion lines and the gluons are denoted by the helical lines. 


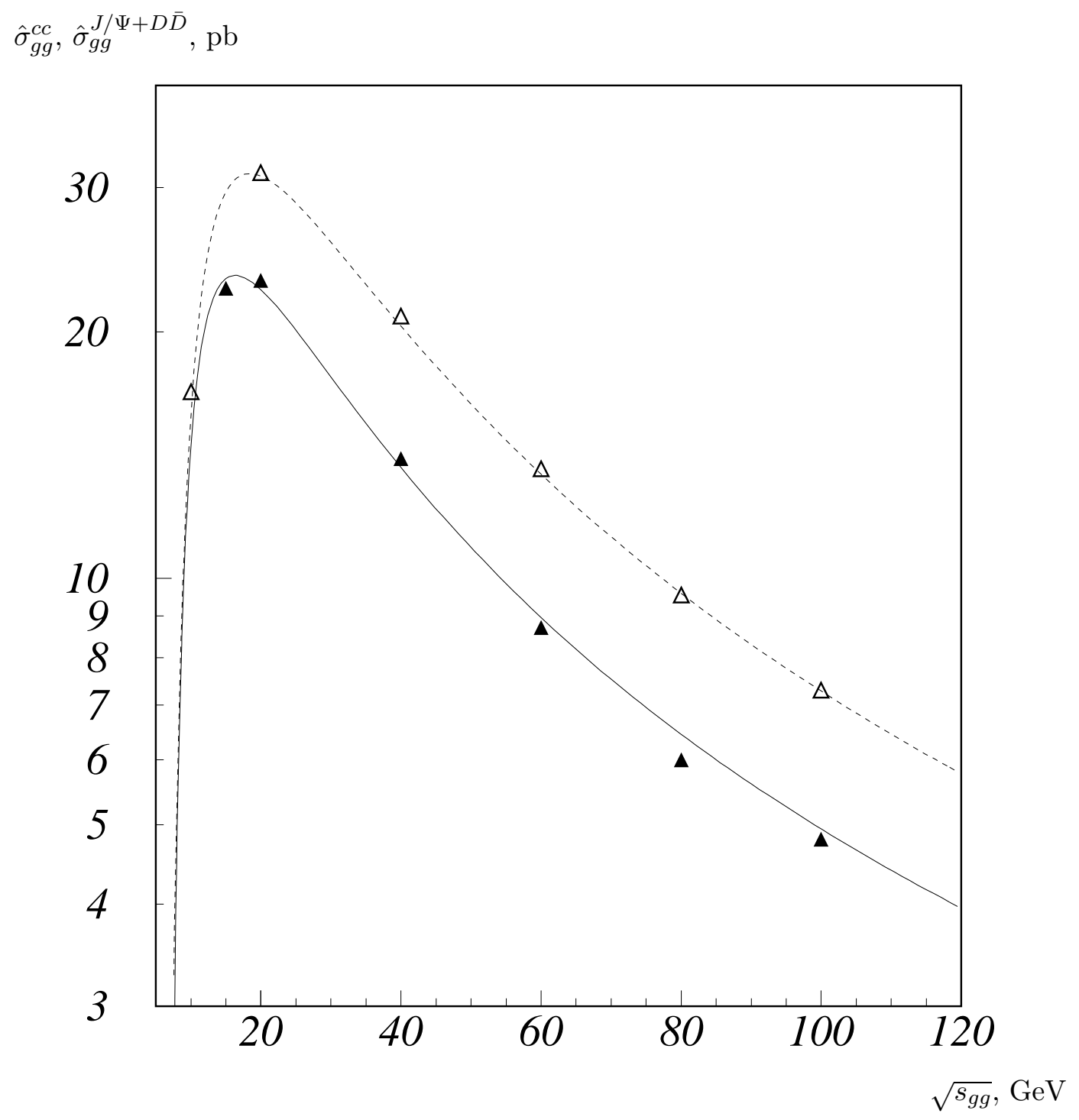

FIG. 2. The total cross-section of the gluon-gluon production of (cc)-diquark (solid triangle) and $J / \Psi+D \bar{D}$ (empty triangle) in comparison with the approximations of (8) and (10) (solid and dashed curves, correspondingly). 


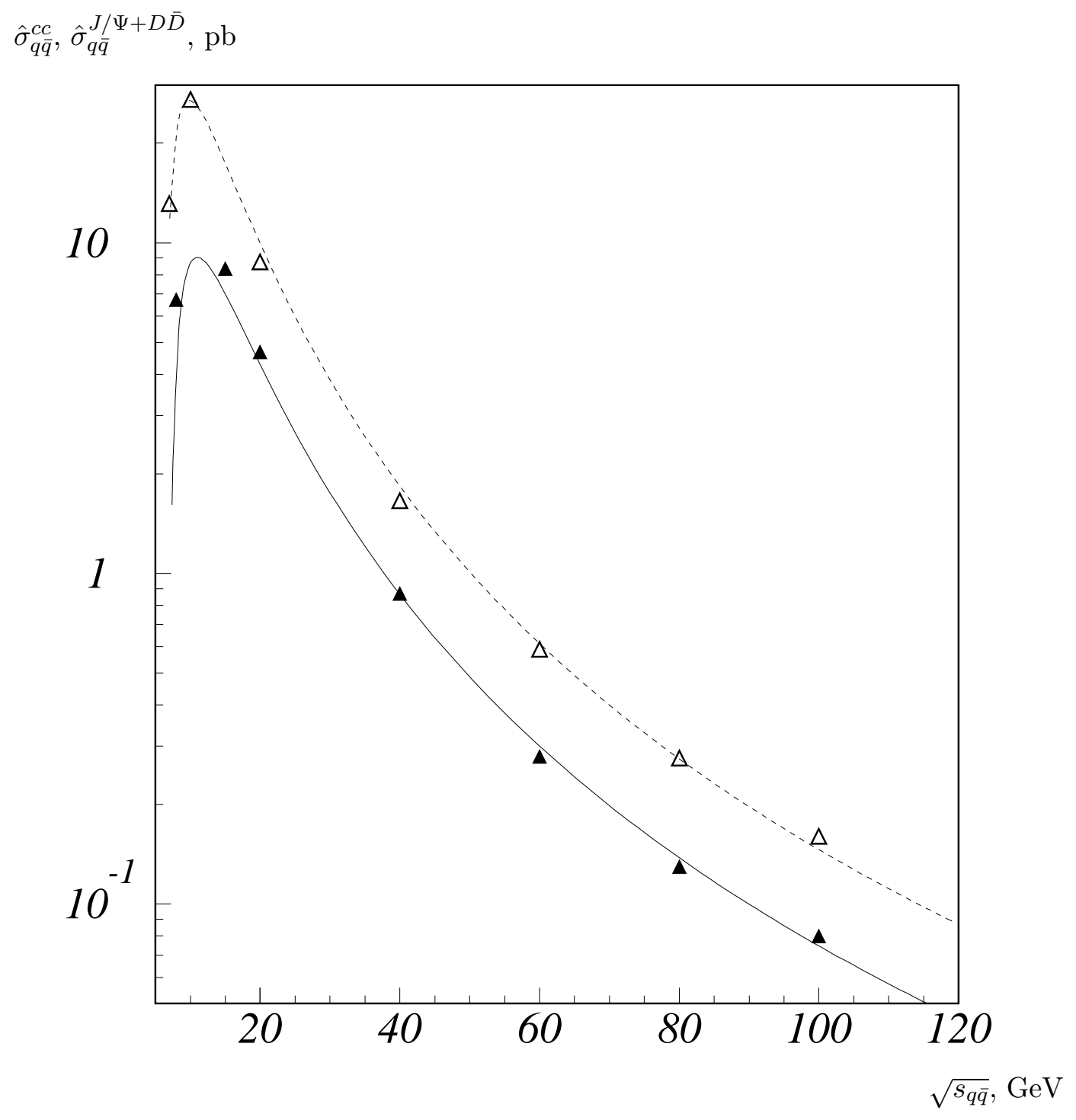

FIG. 3. The total cross-section of the quark-antiquark production of (cc)-diquark (solid triangle) and $J / \Psi+D \bar{D}$ (empty triangle) in comparison with the approximations of (9) and (11) (solid and dashed curves, correspondingly). 


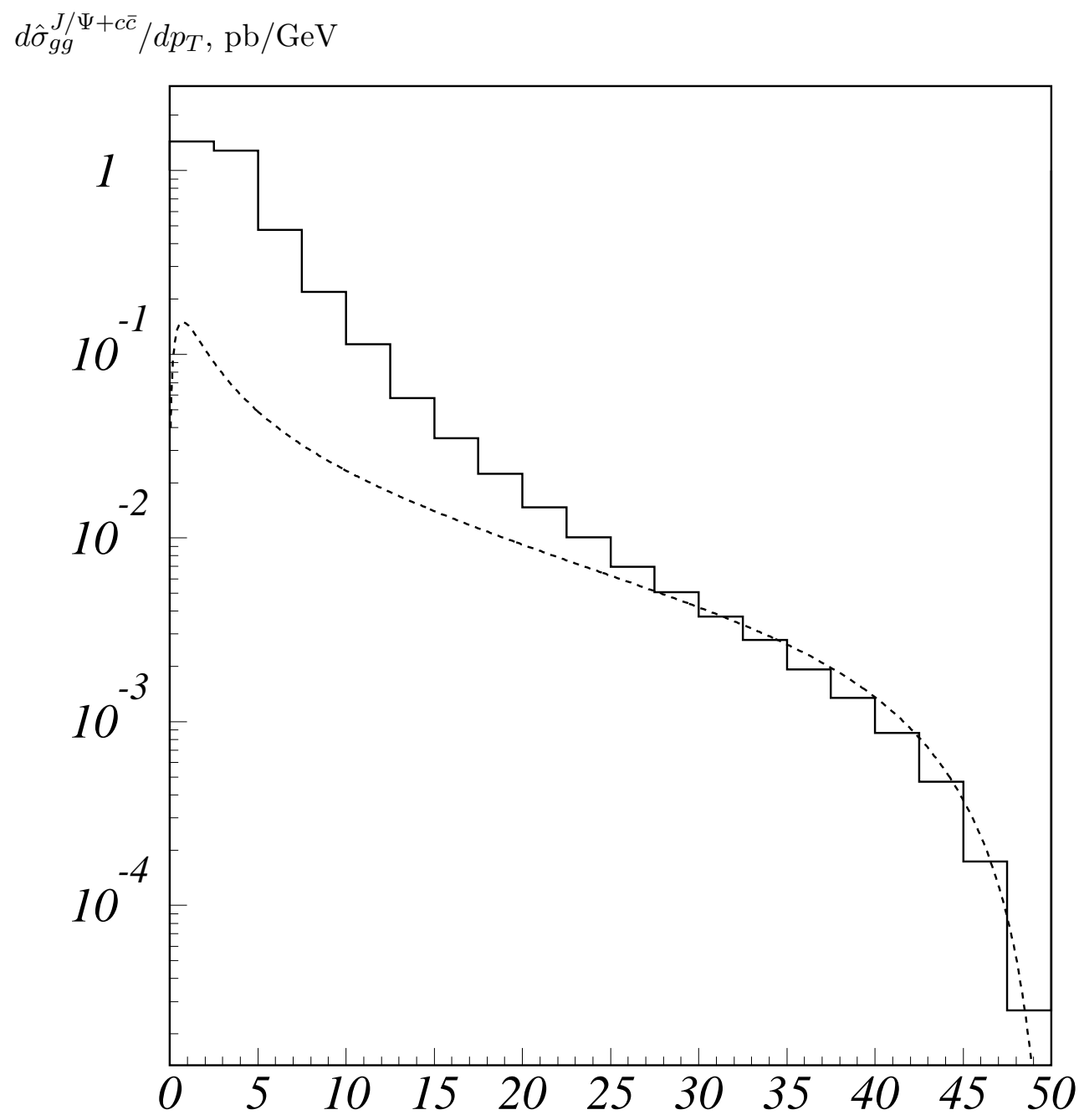

$$
p_{T}, \mathrm{GeV}
$$

FIG. 4. The differential cross-section for the associated production of $J / \Psi+c \bar{c}$ in the gluon-gluon subprocess at $100 \mathrm{GeV}$ (solid histogram) in comparison with the prediction of fragmentation model (dashed curve), correspondingly. 
$\sigma_{\pi^{-} p}^{c c}, \hat{\sigma}_{\pi^{-} p}^{J / \Psi+D \bar{D}}, \mathrm{nb}$

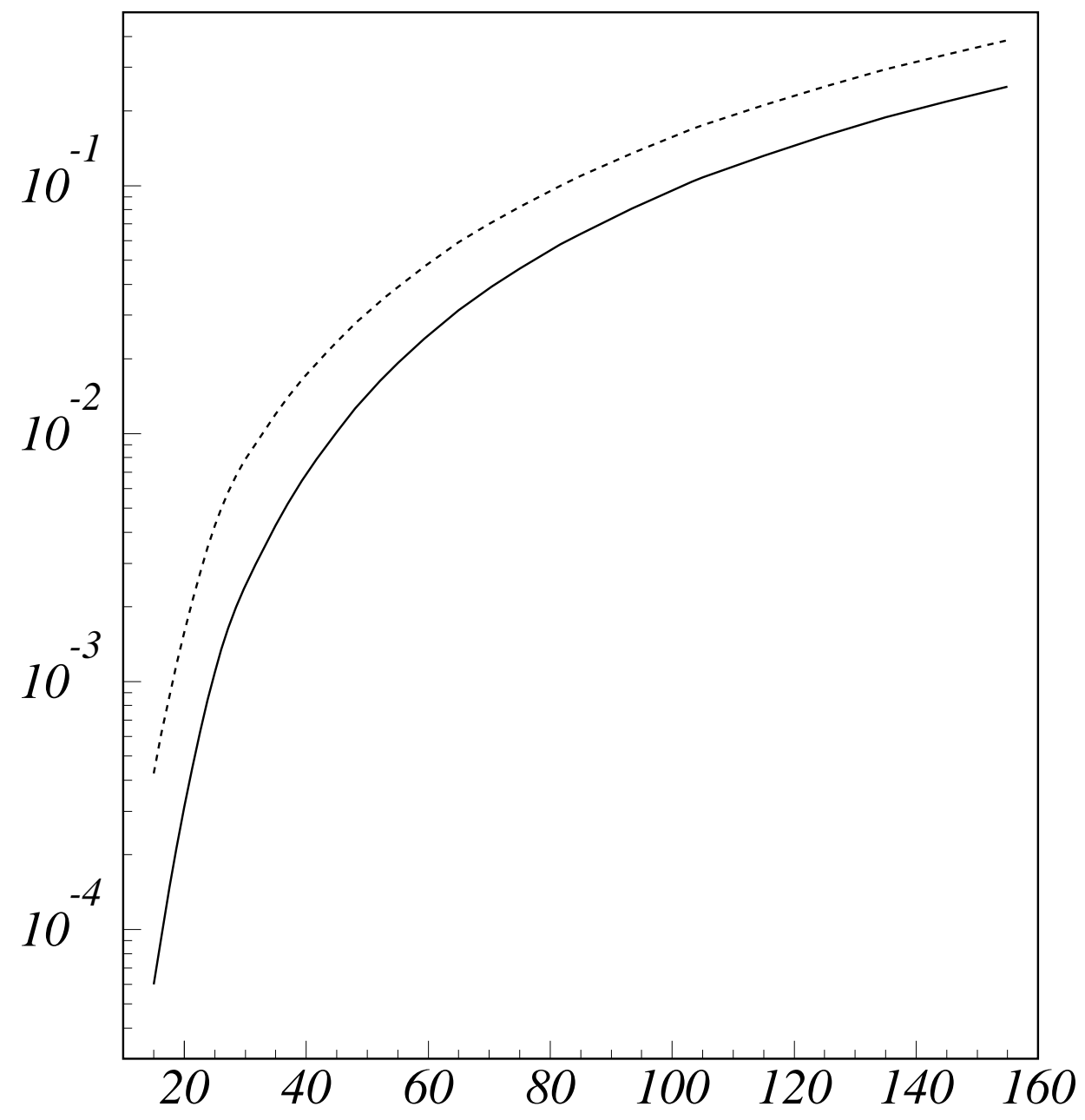

$\sqrt{s_{\pi^{-}}}, \mathrm{GeV}$

FIG. 5. The total cross-section of the pion-proton production of $(c c)$-diquark and $J / \Psi+D \bar{D}$ (solid and dashed curves, correspondingly). 


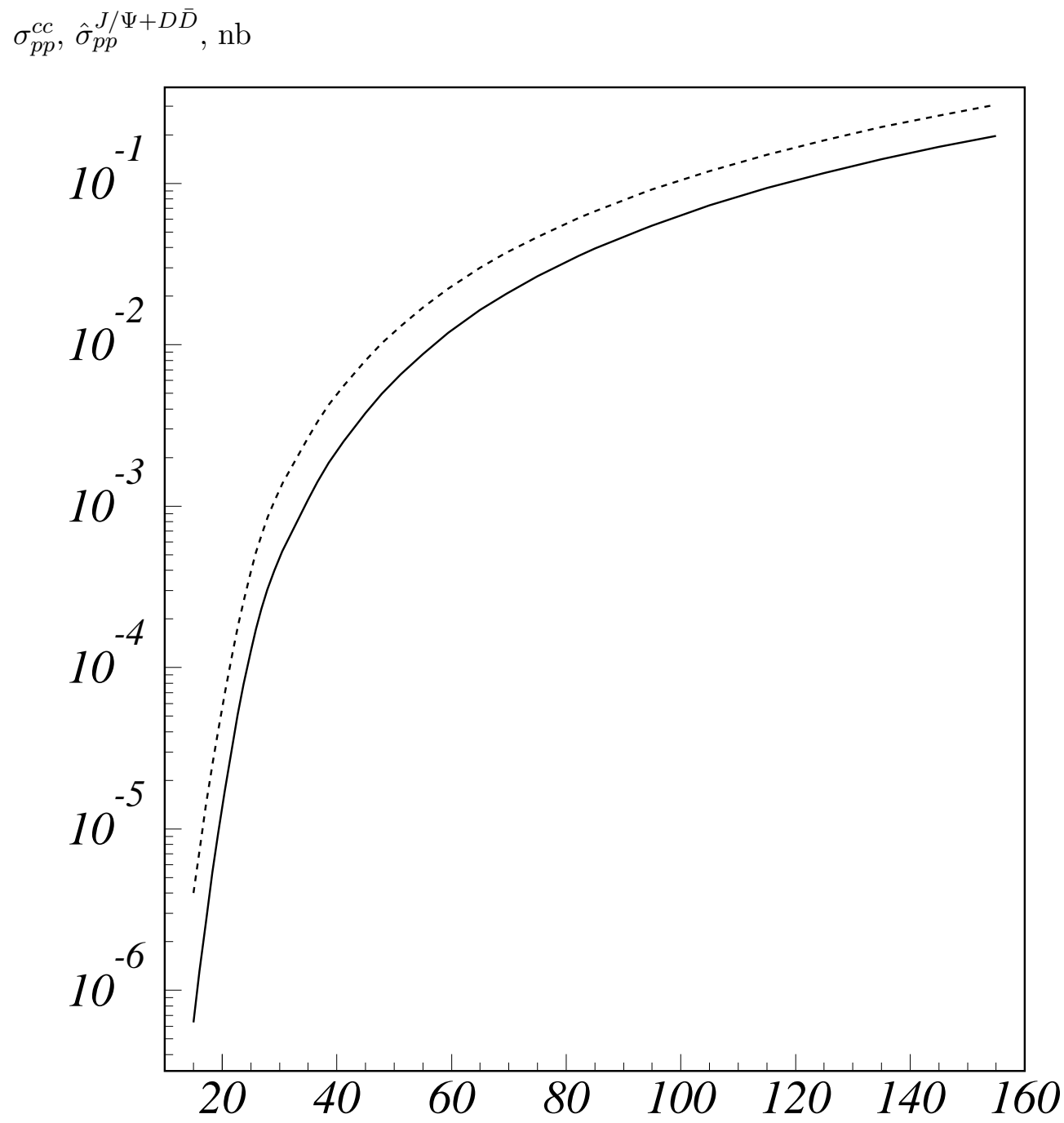

$$
\sqrt{s_{p p}}, \mathrm{GeV}
$$

FIG. 6. The total cross-section of the proton-proton production of $(c c)$-diquark and $J / \Psi+D \bar{D}$ (solid and dashed curves, correspondingly). 
$d \sigma_{\pi^{-} p}^{c c} / d p_{T}, d \sigma_{\pi^{-} p}^{J / \Psi+D \bar{D}} / d p_{T}, \mathrm{pb} / \mathrm{GeV}$

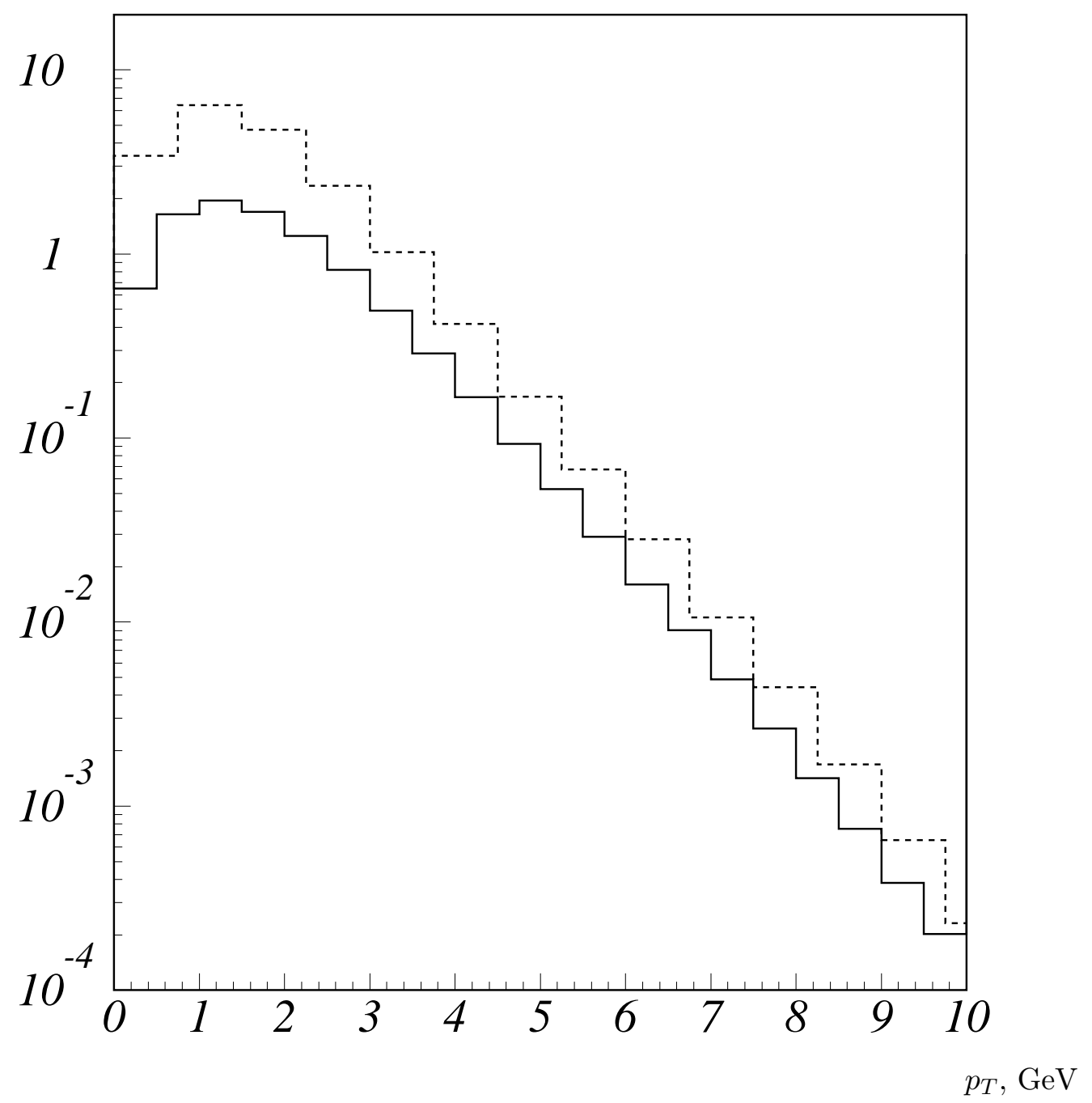

FIG. 7. $\quad d \sigma_{\pi^{-} p}^{c c} / d p_{T}$ (solid histogram) and $d \sigma_{\pi^{-} p}^{J / \Psi+D \bar{D}} / d p_{T}$ (dashed histogram) at the pion-proton interaction energy of $35 \mathrm{GeV}$. 


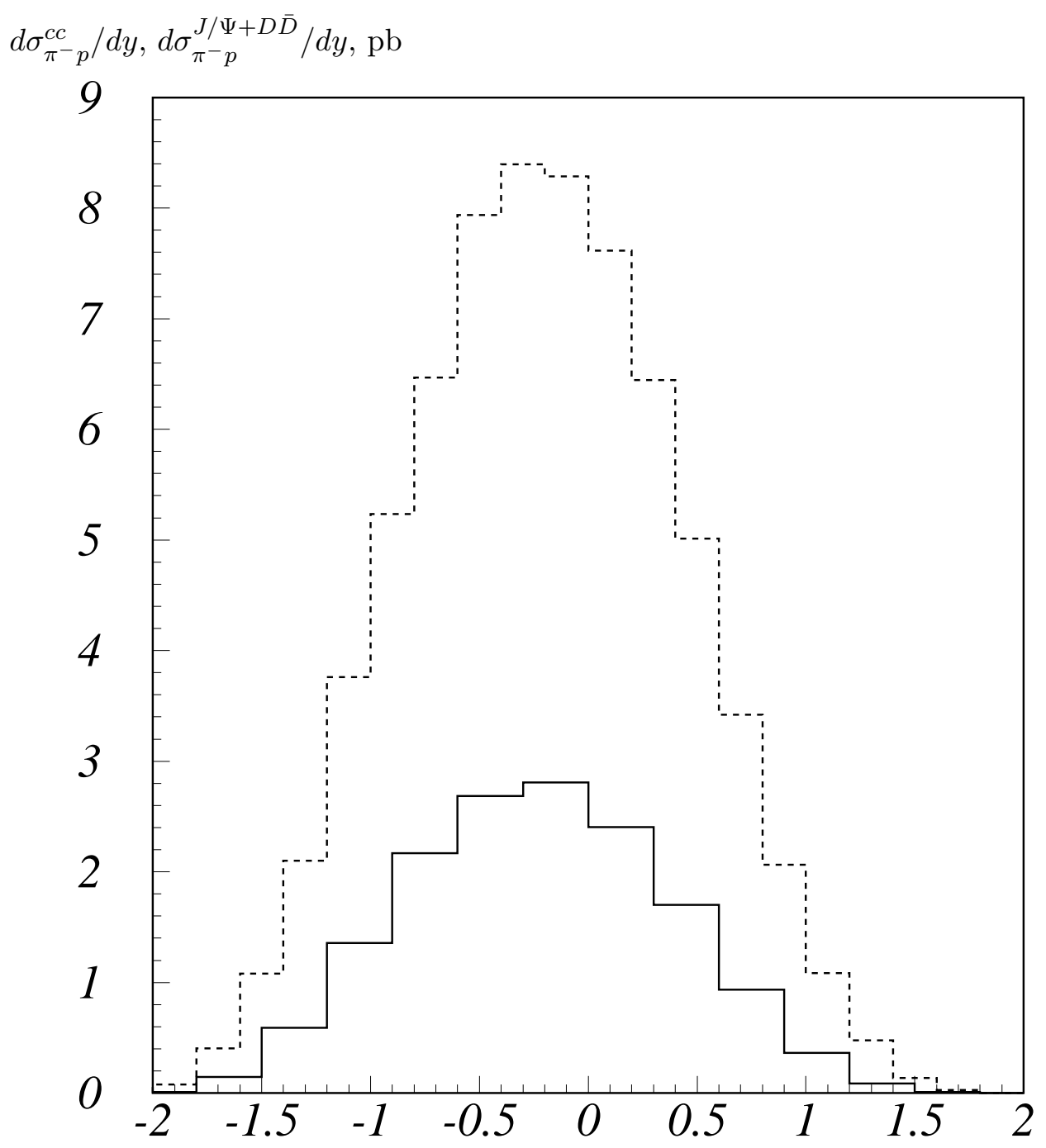

FIG. 8. $d \sigma_{\pi^{-} p}^{c c} / d y$ (solid histogram) $d \sigma_{\pi^{-} p}^{J / \Psi+D \bar{D}} / d y$ (dashed histogram) at the pion-proton interaction energy of $35 \mathrm{GeV}$. 


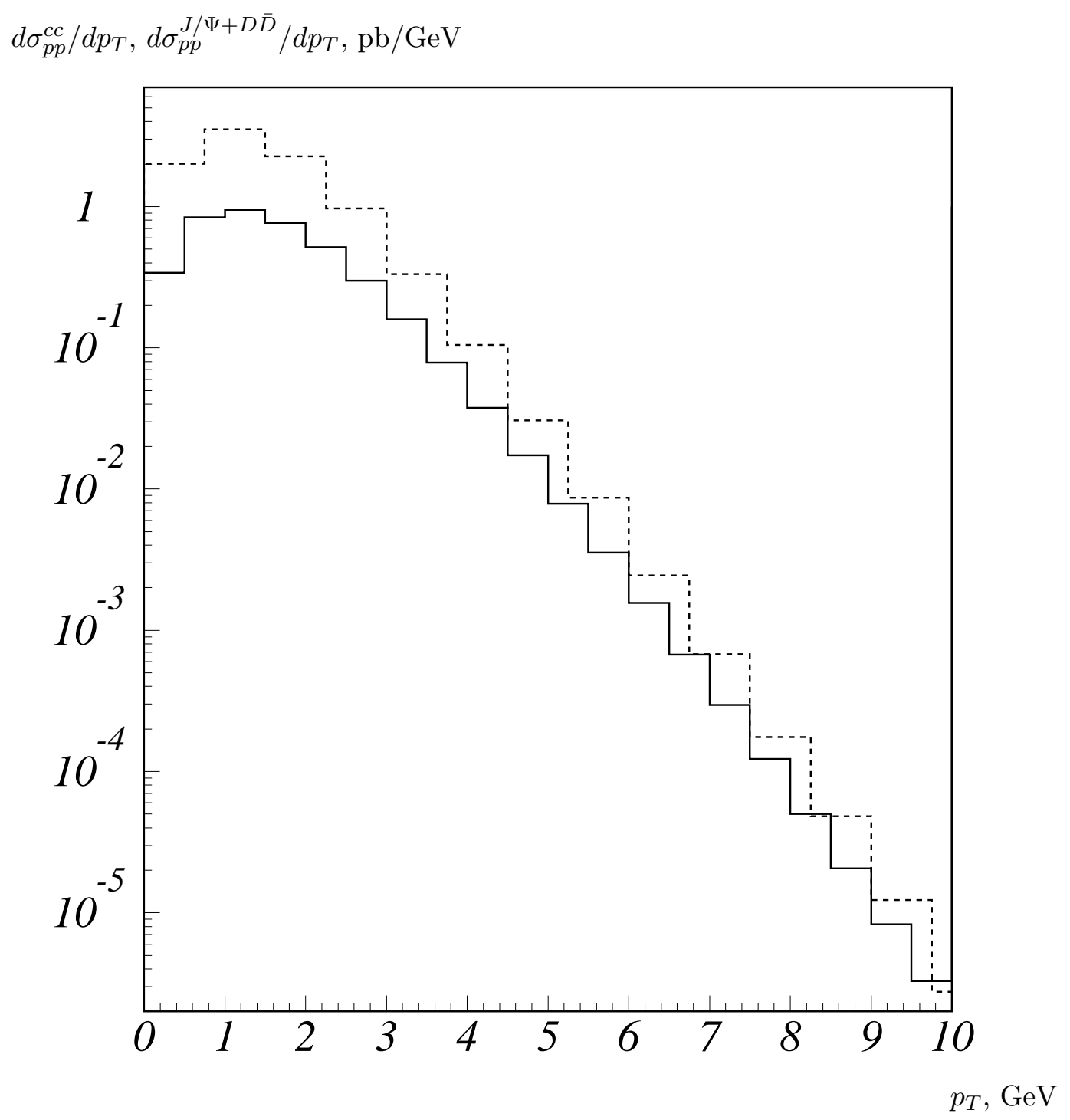

FIG. 9. $\quad d \sigma_{p p}^{c c} / d p_{T}$ (solid histogram) and $d \sigma_{p p}^{J / \Psi+D \bar{D}} / d p_{T}$ (dashed histogram) at the proton-proton interaction energy of $40 \mathrm{GeV}$. 


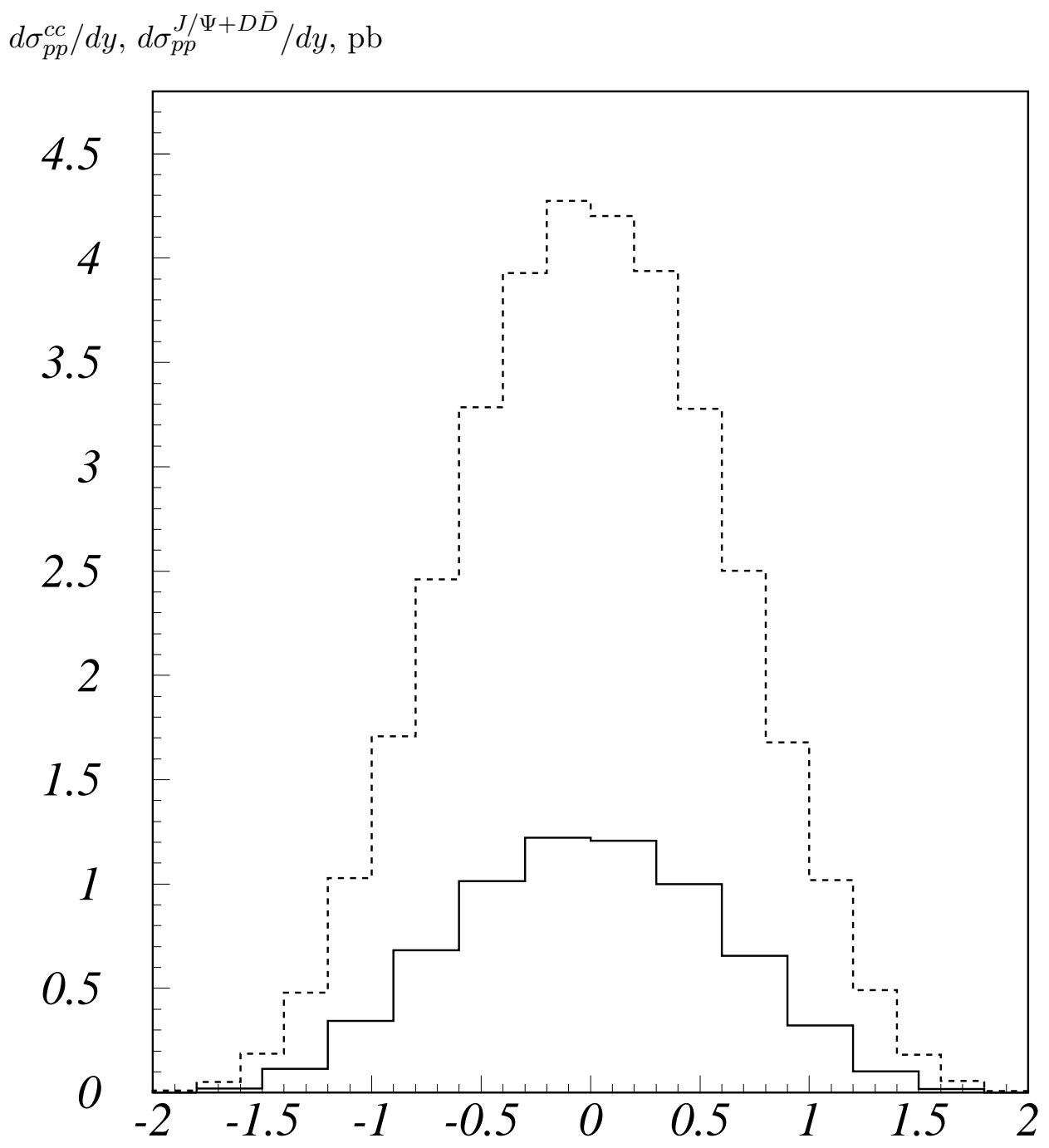

y

FIG. 10. $\quad d \sigma_{\pi^{-} p}^{c c} / d y$ (solid histogram) $d \sigma_{\pi^{-} p}^{J / \Psi+D \bar{D}} / d y$ (dashed histogram) at the proton-proton interaction energy of $40 \mathrm{GeV}$. 
$d \sigma_{p p}^{\Xi_{c c}^{(*)}} / d p_{T}, d \sigma_{p p}^{J / \Psi+D \bar{D}} / d p_{T}, \mathrm{nb} / \mathrm{GeV}$

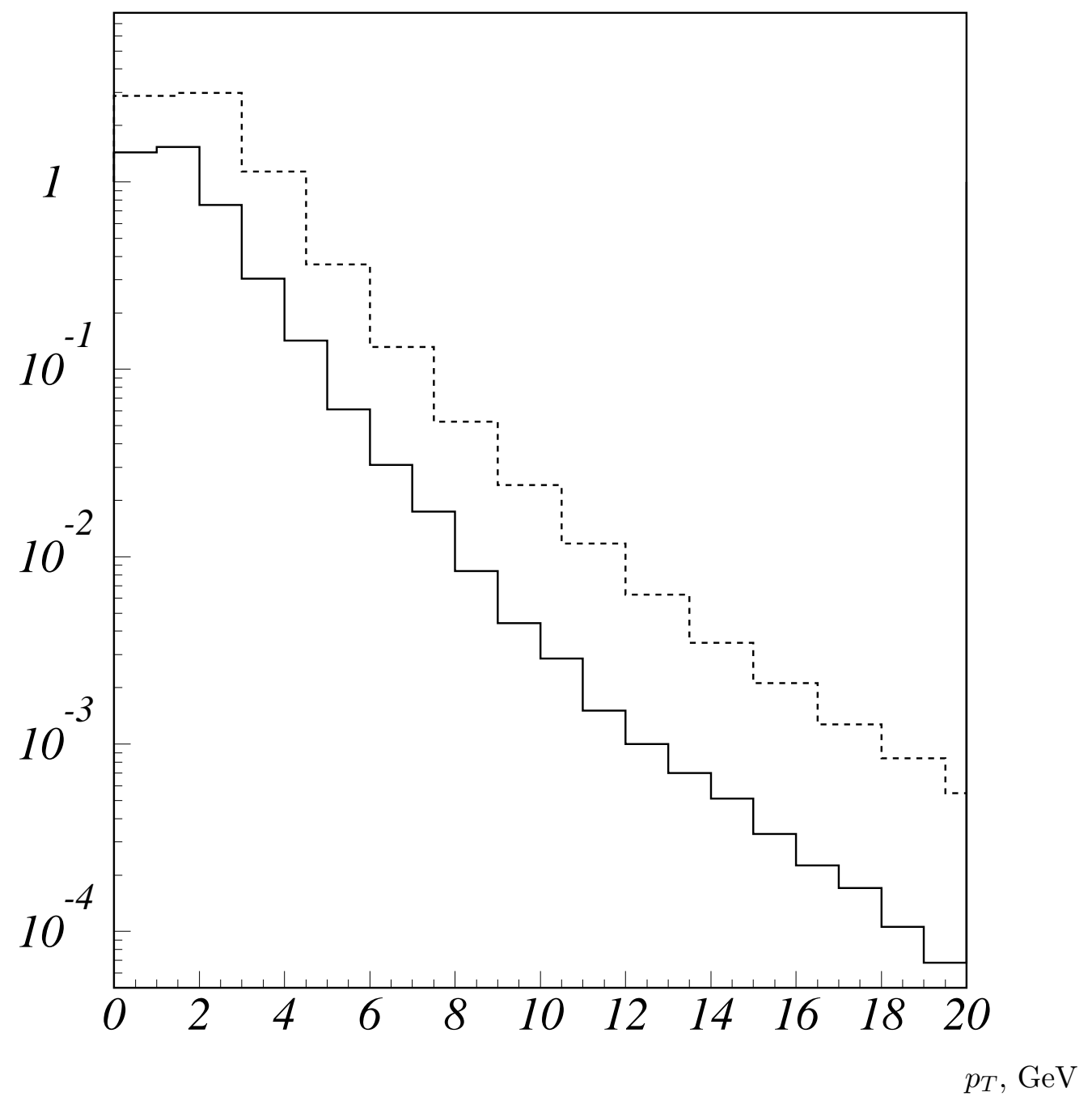

FIG. 11. $d \sigma_{p p}^{\Xi_{c c}^{(*)}} / d p_{T}$ with taking into account the fragmentation of $c c$-diquark into $\Xi_{c c}^{(*)}$-baryon (solid histogram) and $d \sigma_{p p}^{J / \Psi+D \bar{D}} / d p_{T}$ (dashed histogram) at proton-proton interaction energy of $1.8 \mathrm{TeV}$. 
$d \sigma_{p p}^{\Xi_{c c}^{(*)}} / d p_{T}, d \sigma_{p p}^{J / \Psi+D \bar{D}} / d p_{T}, \mathrm{nb} / \mathrm{GeV}$

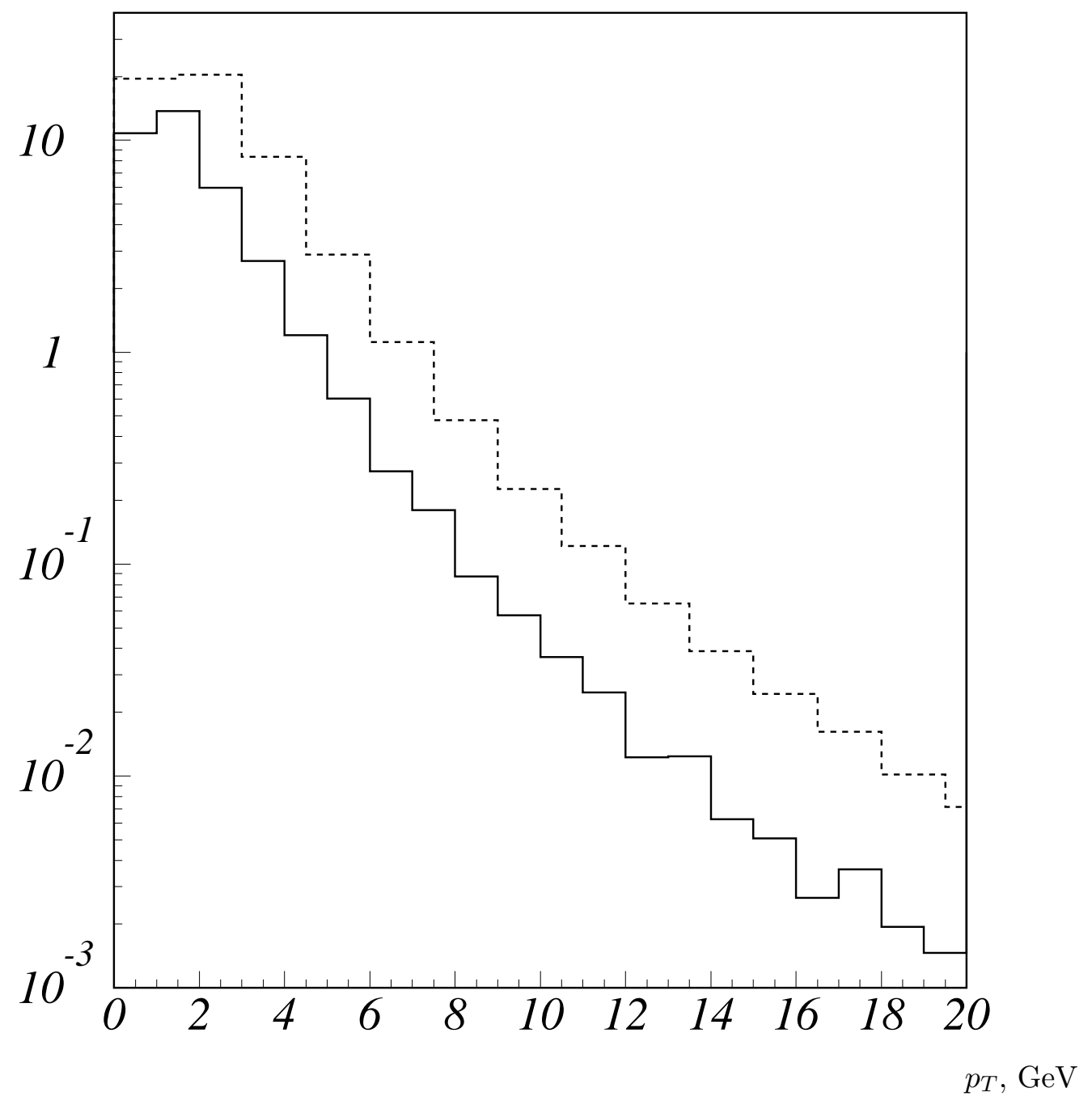

FIG. 12. $\quad d \sigma_{p p}^{\Xi_{c}^{(*)}} / d p_{T}$ with taking into account the fragmentation of $c c$-diquark into $\Xi_{c c}^{(*)}$-baryon (solid histogram) and $d \sigma_{p p}^{J / \Psi+D \bar{D}} / d p_{T}$ (dashed histogram) at proton-proton interaction energy of $14 \mathrm{TeV}$. 